\title{
Teaching OOP in C++ Using an Artificial Life Framework
}

\author{
Richard E. Pattis \\ Computer Science Department \\ Carnegie Mellon University \\ Pittsburgh, PA 15213-3891 \\ pattis@acm.org
}

\begin{abstract}
This paper explores the framework method for teaching object-oriented programming. Specifically, it describes a hierarchy of $\mathrm{C}++$ classes that implement the framework for an Artificial Life simulator. Students learn how to read these classes and extend them via inheritance: they design and implement subclasses that encapsulate the behavior and state of environments and the entities that inhabit them. The simulator constructs an artificial world from objects of these subclasses; then, it animates these objects as they interact during the simulation. This paper includes one sample project and both subclasses that implement its solution.
\end{abstract}

\section{1: Introduction}

A framework comprises application-specific code that programmers extend to build complete applications. In an object-oriented programming (OOP) framework, this code is encapsulated in a hierarchy of classes; the interactions among the objects constructed from these classes map out the basic structure of the application. Programmers typically extend such an OOP framework by deriving specialized subclasses from the general classes already in its hierarchy.

In the framework method of teaching OOP, students learn how to read and extend some OOP framework by building various applications from it. First they learn how to read and understand the classes (their public, procedural interfaces) in the inheritance hierarchy of the framework. Then students learn how to write their own subclasses, which can store local state and can override old (and define new) behavior. ${ }^{\dagger}$

A carefully designed framework provides an exemplary, concrete context in which students can acquire programming and problem solving skills. Students can solve interesting and sophisticated programming problems by making only small extensions to such a framework. Typically, a framework used for teaching will include an important visual component that continuously displays information about the state of the computation, supplying feedback to students concerning the correctness of their executing code.

Brown University [Conner95] teaches OOP early in CS1 using a Graphical User Interface framework named GP (Graphics Package). Students extend GP's classes to build various Windows ${ }^{\mathrm{TM}}$ applications by using Borland Pascal. ${ }^{\mathrm{TM}}$

fOther methods of teaching OOP are more bottom-up: students study control/data structures first, then classes, finally inheritance.

Permission to make digital/hard copy of part or all of this work for personal or classroom use is granted without fee provided that copies are not made or distributed for profit or commercial advantage, the copyright notice, the title of the publication and its date appear, and notice is given that copying is by permission of ACM, inc. To copy othenwise, to republish, to post on servers, or to redistribute to lists, requires prior specific permission and/or a $10 \theta$

SIGCSE ' 97 CA, USA

(6) 1997 ACM 0-89791-889-4/97/0002...\$3.50

\section{2: Get A-Life: An Artificial Life Framework}

"Artificial Life (A-Life) is the study of synthetic systems that exhibit behaviors that are characteristic of natural living systems" [Langton89]. A-Life simulations provide both an interesting and motivational context in which students can learn both the concepts and mechanics of $O O P$ naturally.

Get A-Life is the name of a package that is designed to be used during the first few weeks of any course that teaches $O O P$ in $C++$. The Get A-Life package includes

(1) an A-Life simulation framework implemented as a hierarchy of $\mathrm{C}++$ classes, whose interfaces are carefully formatted and extensively documented via comments.

(2) a short textbook [Pattis97] explaining this framework, and the OOP concepts and $\mathrm{C}++$ language features that students need to read, understand, and extend it - to write their own A-Life application programs.

This combination provides an apt vehicle for jumpstarting students into their study of OOP and $\mathrm{C}++$.

Students extend the Get A-Life framework by using inheritance to derive new classes that model environments and entities. They override/define new behavior for these classes by writing a collection of small member functions that operate on their local/class data members. Then, the simulator uses these classes to construct an artificial world composed of environment and entity objects, and animates their behavior as they interact with each other. Furthermore, programmer-chosen observers collect and display, in realtime, statistical information about the simulation.

As students learn to read and extend the Get A-Life framework, they quickly acquire many basic OOP/C+ skills: how to

- read class declarations in an inheritance hierarchy

- write derived classes encapsulating behavior and state

- override/define new member functions

- define object and class data members

- call application-specific generators and functional iterators

- instantiate simple template classes/functions

- catch exceptions

all while programming a variety of structurally similar A-Life simulations. The Get A-Life textbook fully analyzes about half a dozen of these simulations, and includes dozens of others as projects: 1-d/2-d cellular automata; animals evolving/flocking/ foraging/hunting/stockpiling; physics models; video games; image processing, parallel computing etc. [Dewdney88, 90, 93, Garcia-Ruiz93, Gaylord95, Resnick94, and Rucker93].

Finally, as students implement derived subclasses, they also learn more standard programming material: functions that use parameters, simplc control structures, primitive data types, expressions containing built-in operators, and other "low-level" $\mathrm{C}++$ language features. 


\section{1: A Get A-Life Application}

The Get A-Life framework builds applications (see Figure 1) in which the environment and entity objects display themselves in a map that occupies the left portion of the main Get $\mathrm{A}$-Iife window (partly obscured below by the Run menu). The total elapsed simulated time appears in the upper-right corner of this window too. All the observer objects initially display themselves in their own windows at the right hand of the Get A-Life window; of course, users can move, resize, and minimize any window to their taste.

Users can run the application from a standard or random initial configuration. They can also control the application as it runs by selecting various options. An application continues running until its termination condition (via an object supplied to the simulation's constructor) is met, the user manually terminates it, or it runs out of resources. In any of these cases, the user can then exit or rerun the application.

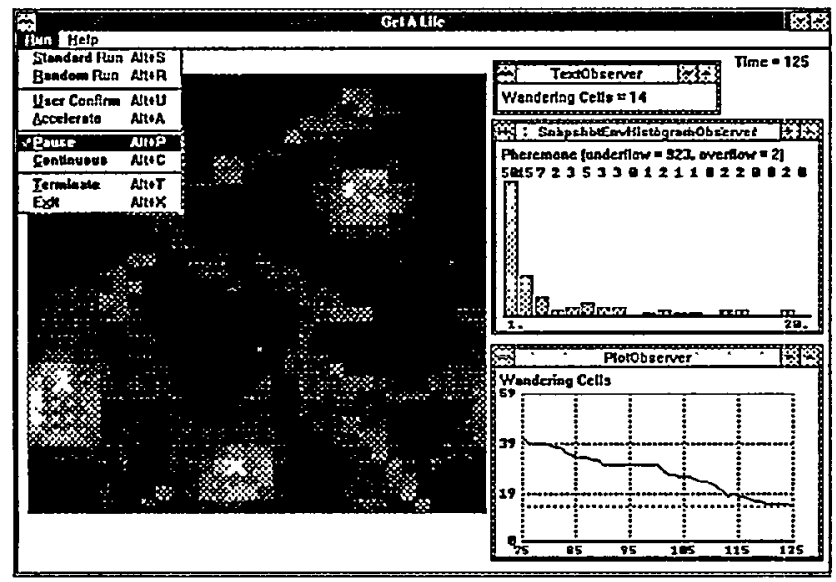

Figure 1: The Get A-Life Application and Observer Windows

\section{2: The Main Get A-Life Class Hierarchy}

The Get A-Life framework comprises about fifty classes arranged in over a dozen inheritance hierarchies. The most important hierarchy is based on the simulton class, which is shown in Figure 2. It is an abstract base class that is the root for the environment and entity hierarchies.

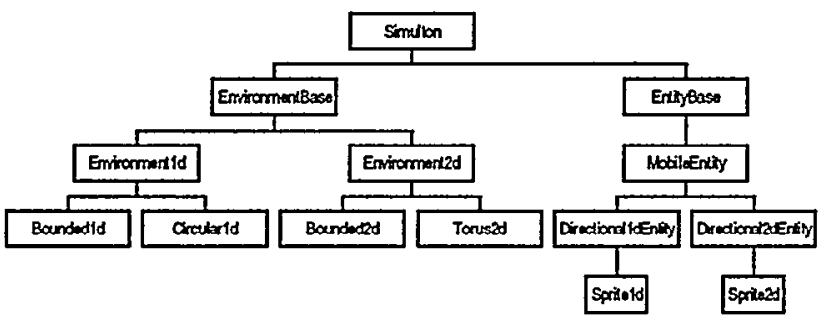

Figure 2: Simulton Class Hierarchy

Environments are arranged in a fixed topology chosen by the programmer. Entities are mobile within these environments, and they can be created and destroyed dynamically during a simulation. Environments and entities can override the do-nothing behavior of their Initialize, Plan, and Act member functions, which they inherit from their simulton ancestor class. They must override their Display function, which is declared as a pure virtual function in simuiton.
Other useful, but less important class hierarchies include those for observers (e.g., displaying values, extremal values, histograms, and plots), icons (used by the environment and entity objects to display themselves), and coordinates.

\section{3: The Hardwired Get A-Life Control Structure}

The control structure for the Get A-Life framework is a simple, fixed loop that is common to all applications:

(1) Construct: Construct an A-Life simulator, its world, and all its observers: specify the world's size, a genesis function (for constructing environment objects at each location in the world), and a termination object.

(2) Initialize: (Re)set the time. Execute the Initlalize member function of each environment object: initialize its state and construct the initial entities that inhabit it.

(3) Observe: Execute the Observe member function of each observer object, which collects data to display.

(4) Display: Display the time. Execute the Dioplay member function of each environment object and all the entity objects that inhabit it; combine, assemble, and display the returned icons in the form of a map. Then, executc the Display member function for each observer object.

(5) Terminate?: Terminate the simulation if the Iapiniahed member function of the simulator's termination object (supplied at construction time) returns true.

(6) Plan: Advance the time. Execute the Rlan member function of each environment, and then each entity object.

(7) Act: Execute the Act member function of cach environment and then each entity object; randomize the order, so no object is given a consistent temporal advantage.

(8) Loop: Repeat execution at step (3).

\section{4: Extending the Get A-Life Framework}

Once students learn enough details about Get A-Life's class hierarchies and the $\mathrm{C}++$ language features needed to derive classes, they can begin writing their own applications.

(1) Declare the application's environment and entity subclasses, derived from the best/closest available base classes. These might be classes in the framework, classes previously written by the student, or instructorsupplied classes meant to help students begin a project.

(2) Declare the required local state for each environment and entity class by using built-in $\mathrm{C}++$ data types (int, double, and bool) and other concrete classes provided by the framework (Counter, colorshapeIcon, etc.).

(3) Define the required behavior for each subclass by overriding old/defining new member functions by using simple $\mathrm{C}++$ statements: function calls, assignment statements, and conditional statements -all of which can contain arithmetic and logical expressions.

\section{3: Generators and Iterators}

Get A-Life applications involve no explicit iteration over compound data structures. To understand how simulations can be written without these language features, we must examine the interaction between generators and iterators. Basically, generators generate a collection of simultons (either environment or entity objects), and iterators process such collections of objects, often under the control of another function that is passed as a parameter to the itcrator. 


\section{1: Generators}

Table 1 specifies some of the built-in Get A-Life generators.

\begin{tabular}{|c|}
\hline $\begin{array}{l}\text { Table 1: Generator Functions } \\
\text { when forming names, [] means optional and I means choice }\end{array}$ \\
\hline Envi ronmentrists 211 [Other] Enpironments () \\
\hline Ent1 tyutste 211 [Other|Local [NonLocal] Ent1ties () \\
\hline 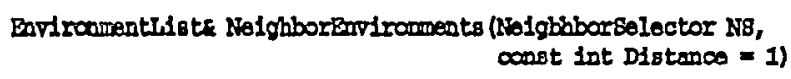 \\
\hline $\begin{array}{r}\text { EntityListe Noighborentities (NeigbhborEelector NS, } \\
\text { canst int Distance = 1) }\end{array}$ \\
\hline
\end{tabular}

For example, a simulton calling Al1otherEnvironments () generates a list of all environments except the one occupied by that simulton; a simulton calling Alluocalentities() generates a list of all entities in that simulton's environment.

The last two Nelghbor generators require two parameters: a Ne1ghborselector function and a distance over which to apply it: a distance of $\mathrm{a}$ includes neighbors up to $\mathrm{z}$ units away in any dimension. Table 2 specifies the built-in neighborhood selector functions. Programmers can also write their own selectors: each is just a predicate that returns true whenever the Candidate parameter belongs in the neighborhood of the Center parameter.

Table 2: Neighbor Selectors

when forming names, I means choice

typedef bool (Nolghborselector)

(canst simultone center, const simultone candidate);

Prefixesi all | Cross | I | Hordzontal | Vertical

Sufixes; Inclusive | Exclugive

In a 2D Topology, with Distance $=1$

\begin{tabular}{|c|c|c|}
\hline$X$ & $V$ & $X$ \\
\hline$H$ & $C$ & $H$ \\
\hline$X$ & $V$ & $X$ \\
\hline
\end{tabular}

$C$ in Inclusive

$H$ in $A 11$, Cross, Horizontal

$\mathrm{V}$ in $\mathrm{A11}$, Cross, Vertical

$X$ in $A 11, X$

For example, a simulton calling

NeighborEnvironments (AllNeighborsExclusive)

would generate all 8 environments immediately surrounding, but not including, the environment occupied by the simulton (here the Distance defaults to 1 ).

\section{2: Iterators}

Table 3 specifies some of the built-in Get A-Life iterators. All these functions require a simulton list (see the generators above) as a parameter. Those including Satiafy also require a boolean member function as a second parameter; also, the Gradlent functions (which are templates) require a second parameter that is a member function that returns a result whose value can be relationally compared with another value of the same type; typically, such member functions return some standard numeric type, such as int or double.
Table 3: Functional Iterators

(Counters, Selectors, Filters, Gradients)

when forming names, [] means optional and I means choice

\begin{tabular}{|c|c|}
\hline Int & Count [ [Not] Batisfy] \\
\hline $\begin{array}{l}\text { Bnvironmente } \\
\text { Entitye }\end{array}$ & $\begin{array}{l}\text { solect [ [Not] Satisfy] } \\
\text { Eelect [ RYot] Bati Bfy] }\end{array}$ \\
\hline $\begin{array}{l}\text { Bardroomentisiste } \\
\text { BntityListe }\end{array}$ & $\begin{array}{l}\text { Bilter [ Not] Batisfy] } \\
\text { B1Iter [ NNot] Batisfy] }\end{array}$ \\
\hline $\begin{array}{l}\text { Burdromente } \\
\text { metitye }\end{array}$ & $\begin{array}{l}\text { Gradient [Haximu|Hinimom] } \\
\text { Gradient [Haximum|Hindram] }\end{array}$ \\
\hline
\end{tabular}

An environment cell in Conway's game of life calling

Countsatisfy

(EConwayent: : Isalive,

NeighborBnvironments (AllNeighborInclusive))

would return a count of the number of all its neighborhood environments that satisfied their IsAlive member function.

A hungry shark entity in a WA-TOR simulation calling

Selectsatisfy

(EWatorint: :IsTuna,

Neighborkntities (CrossNeighborExclusive))

would return a reference to some entity in the requested neighborhood that identified itself by returning true to IsTuna (so the shark could pursue it). If no entity satisfies this predicate, the Selection iterators throw an IteratorFailure exception. An ant in a foraging simulation calling GradiantMaximum

(EAatBnv: :Nestscent, NeighborBnvironments (AllNeighborsinclusive))) would return a reference to the environment in the requested neighborhood whose Nestscent was maximum (so the ant could move back towards its nest with the food it found).

There are other, more special purpose, iterators: e.g., one for adding up numeric quantities and one for evaporating and diffusing (into some neighborhood) percentages of some numeric quantity accumulated in an environment.

\section{4: A Complete Simulation: Cells Forming Colonies}

To understand more concretely the Get A-Life approach to teaching OOP in $\mathrm{C}++$, one must study class declarations in the simulton hierarchy and learn how a few simulations are designed and implemented. This section goes part of the way, presenting one representative programming problem and its solution: simpler simulations may require only an environment class; more complex simulations may require more complicated behavior/state, or even a hierarchy of environment/entity classes. But, it is beyond the scope of this paper to explore in detail the simulton class hierarchy or the methodology for designing/constructing applications.

\section{1: Problem Statement}

A collection of mobile cells need to form themselves into multi-cell colonies. There are no prearranged locations at which cells meet, and there is no "boss" cell that directs the others towards their final locations. Instead, each cell must execute an algorithm that ensures it will eventually form or join some colony. This problem is adapted from the Slime Mold Sporulation problem [Resnick95]. 


\section{2: Entity and Environment Design}

Entities wander around the world (via Reorient and 1 unit movements), secreting and depositing in their environment 1 unit of a special pheromone. ${ }^{\dagger}$ Whenever an entity reaches a location whose pheromone accumulation exceeds 3 units, it stops moving but continues secreting and depositing pheromone there. Entities display themselves as red circles.

Environments are connected on a torus and serve as pheromone repositories. They also evaporate ( $10.0 \%$ total) and diffuse (15.0\% total, to adjacent environments) whatever pheromone they have accumulated. Environments display themselves as green squares, whose intensity is proportional to the amount of pheromone they contain.

The application built from this design appears in Figure 1; but without color, the quality of the information is reduced.

\section{3: Entity and Environment Code}

The $\mathrm{C}++$ code below specifies and implements the entity and environment classes designed above. To save space, comments and special formatting have been removed, and both class declarations have been collected into onc filc, as have both class definitions. First, here is the file colony.h

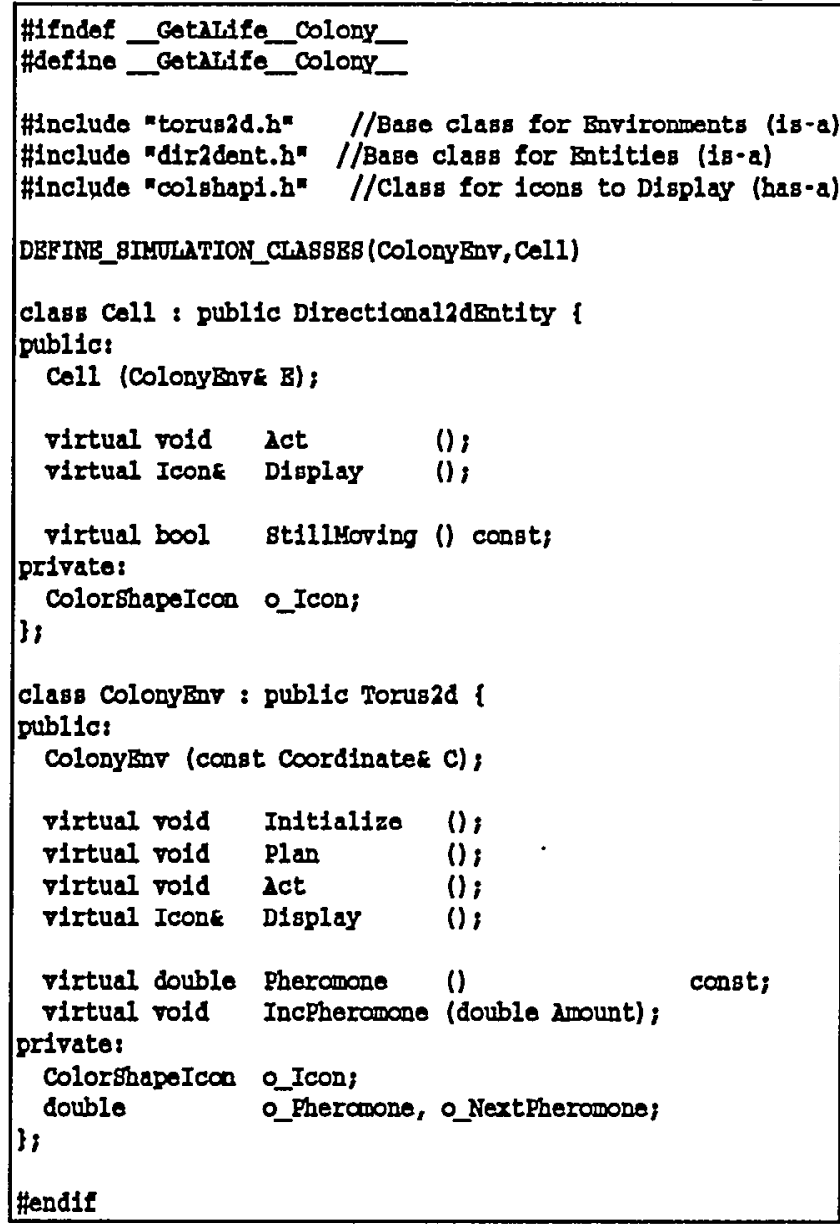

By convention, the prefix $0_{-}$is used for an object's data member, and the prefix $c_{-}$is used for a class's data member

fPhcromones are a chemical substance produced by an animal, serving as a stimulus to other individuals of the same species for one or more behavioral responses. (of which none appear in this code). Read o_Icon as this object's Icon. Next, here is the file colony.cpp

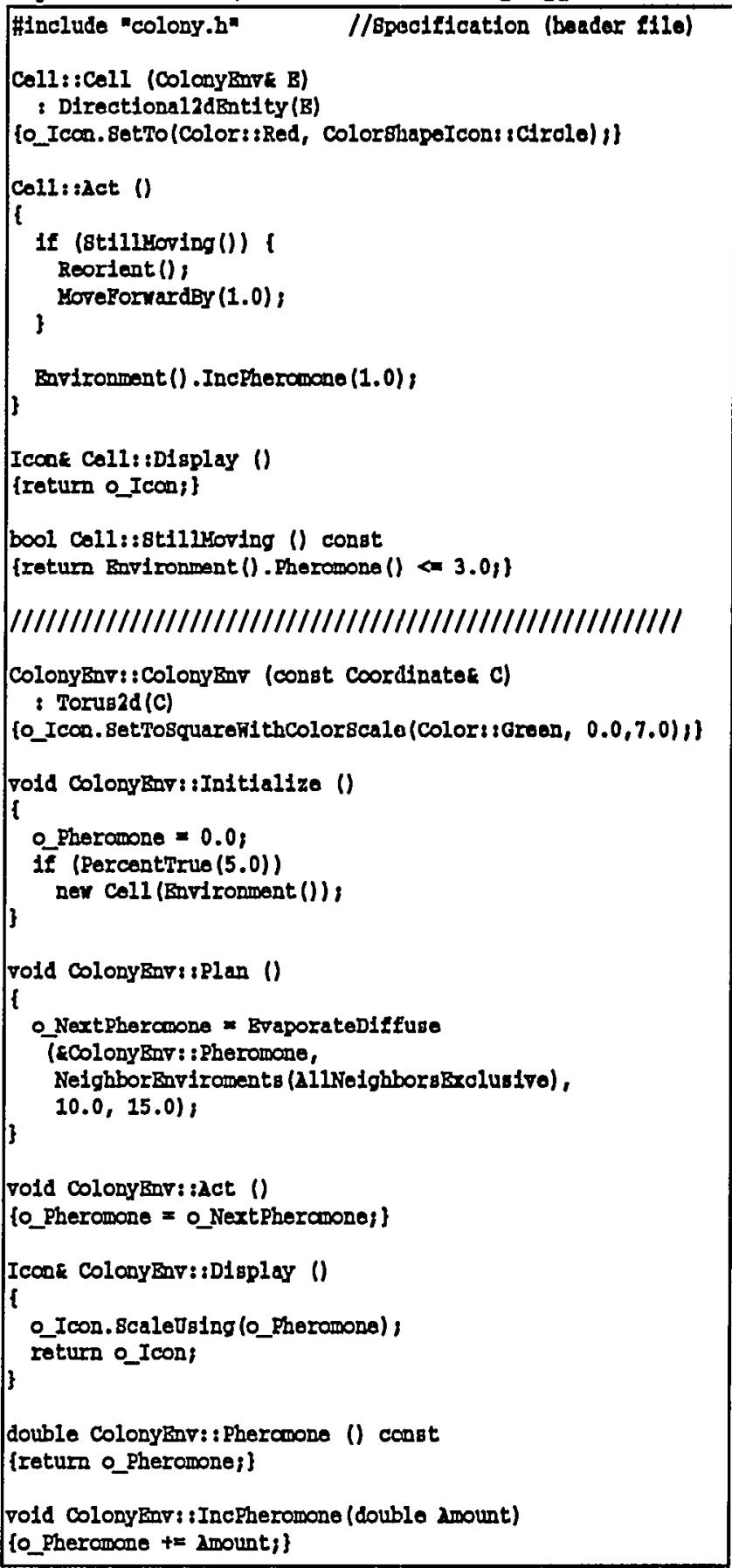

This code illustrates a variety of OOP concepts and $\mathrm{C}++$ language features, including class derivation/inheritance, constructors, overriding and defining new member functions (some with parameters), private data members, assignment statements, if statements, function calls, generators, iterators, and simple relational operators.

Students can easily design and code other simulations, because of the many structural similarities among all the classes that they just write. Once the interfaces and private parts to classes such as these are designed, their member functions are typically small and simple to implement. 
Finally, here is a file with a main program that declares the simulator and its observers, and runs the simulation. It is the file colony. cpp. Instructors may initially write such main programs, to simplify their student's early programming assignments. Notice that the free function wandercount is what the Textobserver and Plotobserver display.

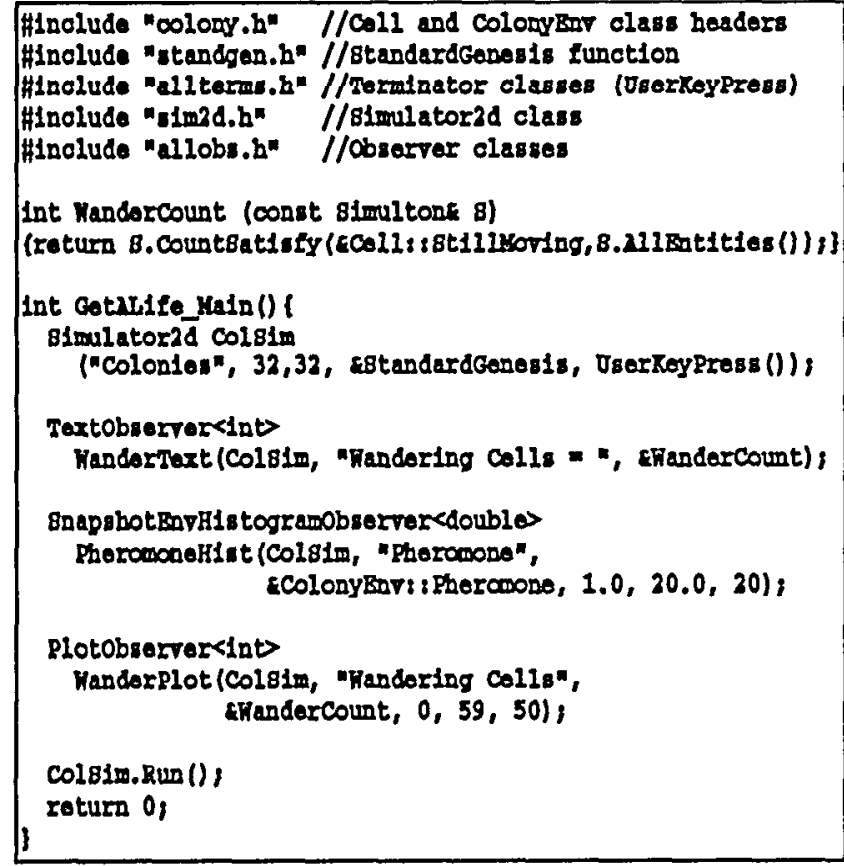

5: Scaleability/Iterative-Enhancement/Documentation

Students arrive in programming courses with a wide range of aptitudes, skills, and experiences. OOP in general, and Get A-Life in particular, allow for many natural ways to scale projects, so different students can reach the same result from different starting points. Instructors can choose these starting points by supplying their students with partially designed/implemented classes. These classes may be semantically incomplete - missing some member functions/ data members; or, they may be just syntactically incomplete -containing member functions that are missing expressions or full statements that the students must supply. In fact, the entirc Get A-Life framework is just one advanced starting point for writing entity-environment simulations.

Once the instructor chooses a starting point, students can design and implement a complete simulation as a sequence of increasingly complex behaviors. Each new behavior results from either modifying an already implemented class, or layering a new class beneath it via class derivation. This iterative-enhancement approach leads to short design/code/ test/debug cycles, where each enhancement produces a new, fully testable (but simplified) application. Such immediate feedback ensures that students follow few blind alleys as they design and implement complicated simulations.

Finally, because OOP heavily promotes code reuse, the students must immediately learn how to read and understand class declarations. From this perspective, students also learn to appreciate good documentation - first as consumers, not producers. Because students must read and then extend class declarations throughout the Get A-Life simulator, all class declarations are carefully formatted and documented via comments. The comment on the declaration of Reorient below appears in the Directional2dBntity class (and is called in Cell: : $\lambda c t)$; other sections in the class declarations document change histories and class invariants.

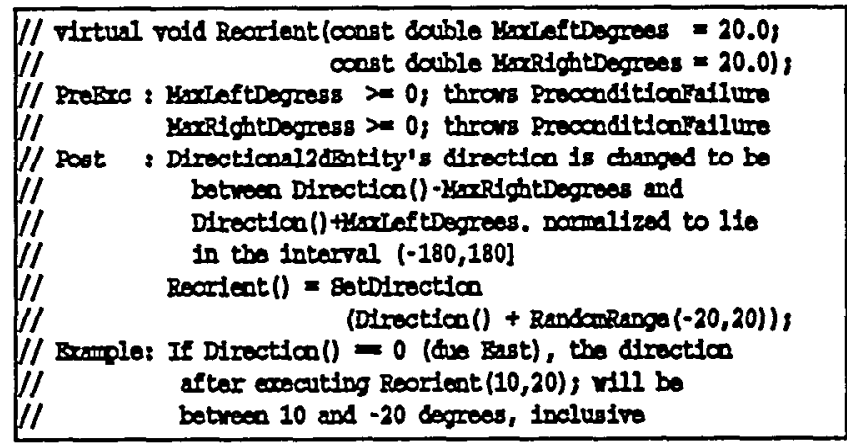

\section{6: Acknowledgments}

Phil Miller delivered an inspiring paper [Meter94] at the SIGCSE'94 conference; he discussed an A-Life simulator that Carnegie Mellon students used at the end of his CS1 course (where they learn explicitly to manipulate linked lists); it was through this work that I began seriously thinking about A-Life as a useful framework for teaching OOP in $\mathrm{C}++$ to novices. Mitchel Resnick's wonderful book [Resnick95] greatly influenced my thinking about A-Life simulations; it continues to be a source of inspiration and contains many interesting A-Life programs that I have rewritten for the Get A-Life framework.

\section{7: Bibliography}

[Conner95] Conner/Niguidula/van Dam, Object Oriented Programming in Pascal: A Graphical Approach, AddisonWesley, 1995

[Dewdney88] A.K. Dewdney, The Armchair Universe: An Exploration of Computer Worlds, Freeman, 1988

[Dewdney90] A.K. Dewdney, The Magic Machine: A Handbook of Computer Sorcery, Freeman, 1990

[Dewdney93] A.K. Dewdney, The Tinkertoy Computer and other machinations, Freeman, 1993

[Epstein96] Epstein/Axtell, Growing Artificial Societies, Brookings Institution Press, 1996

[Garcia-Ruiz93] Garcia-Ruiz, et.al., Growth Patterns in Physical Sciences and Biology, Plenum, 1993

[Gaylord95] Gaylord/Wellin, Computer Simulations with Mathematica, Springer-Verlag, 1995

[Langton89] Christopher G. Langton, Artificial Life I, Addison-Wesley, 1989

[Meter94] Meter/Miller, Engaging Students and Teaching Modern Concepts: Literate, Situated, Object-Oriented Programming, SIGCSE Technical Symposia, 1994

[Pattis97] Richard E. Pattis, Get A-Life: Introducing Object-Oriented Programming in C++ using an Artificial Life Framework, Turing TarPit Press, 1997

[Resnick94] Mitchel Resnick, Turtles, Termites, and Traffic Jams, MIT Press, 1994

[Rucker93] Rudy Rucker, Artificial Life Lab, Waite Group Press, 1993 